\title{
Prevalence and Molecular Characteristics of Carbapenemase-Producing Enterobacteriaceae From Five Hospitals in Korea
}

Seok Hoon Jeong, M.D. ${ }^{1}$, Han-Sung Kim, M.D. ${ }^{2}$, Jae-Seok Kim, M.D. ${ }^{2}$, Dong Hoon Shin, M.D. ${ }^{2}$, Hyun Soo Kim, M.D. ${ }^{2}$, Min-Jeong Park, M.D. ${ }^{2}$, Saeam Shin, M.D. ${ }^{2}$, Jun Sung Hong, B.S. ${ }^{3}$, Seung Soon Lee, M.D. ${ }^{4}$, and Wonkeun Song, M.D. ${ }^{2}$

Department of Laboratory Medicine and Research Institute for Antimicrobial Resistance ${ }^{1}$, Yonsei University College of Medicine, Seoul; Departments of Laboratory Medicine ${ }^{2}$ and Internal Medicine ${ }^{4}$, Hallym University College of Medicine, Chuncheon; Brain Korea 21 PLUS Project for Medical Science ${ }^{3}$, Yonsei University College of Medicine, Seoul, Korea

Background: The emergence of carbapenemase-producing Enterobacteriaceae (CPE) represents a major clinical problem because these bacteria are resistant to most antibiotics. CPE remain relatively uncommon in Korea. We report the prevalence, clinical characteristics, and molecular epidemiology of CPE isolates collected from five university hospitals in Korea.

Methods: Between January and December 2015, 393 non-duplicated isolates that were nonsusceptible to ertapenem were analyzed. Production of carbapenemase, extendedspectrum $\beta$-lactamase, and AmpC $\beta$-lactamase was determined by genotypic tests. Antimicrobial susceptibility profiles were determined by using an Etest. Clonality of Klebsiella pneumoniae carbapenemase (KPC)-2-producing and oxacillinase (OXA)-232-producing Klebsiella pneumoniae isolates was determined by pulsed-field gel electrophoresis (PFGE).

Results: Of the 393 isolates tested, 79 (20.1\%) were CPE. Of these 79 isolates, 47 $(59.5 \%)$ harbored the blaоха-232 gene while the remaining isolates carried genes blakpc-2 $(n=27)$, bla IMP-1 $_{1}(n=4)$, and bla from hospital B, 100\% were resistant to carbapenems, $8 \%$ to colistin, and $0 \%$ to tigecycline. Among the 45 OXA-232 K. pneumoniae at hospital C, 95\% were resistant to ertapenem, $68 \%$ to imipenem, $95 \%$ to meropenem, $10 \%$ to colistin, and $24 \%$ to tigecycline. PFGE analysis revealed a unique pattern for KPC-2 K. pneumoniae and identified 30 isolates belonging to the dominant pulsotypes (PT)1 and PT2 among 41 OXA-232 K. pneumoniae isolates.

Conclusions: CPE strains are present in Korea, with the majority of $K$. pneumoniae isolates producing OXA-232 and KPC-2. The prevalence and predominant genotypes of CPE show hospital-specific differences.

Key Words: Enterobacteriaceae, Klebsiella pneumoniae, KPC-2, OXA-232, Korea
Received: April 26, 2016

Revision received: May 19, 2016

Accepted: June 28, 2016

Corresponding author: Wonkeun Song Department of Laboratory Medicine, Hallym University College of Medicine, Kangnam Sacred Heart Hospital, 1 Shingil-ro, Youngdeungpo-gu, Seoul 07441, Korea Tel: +82-2-829-5259

Fax: +82-2-847-2403

E-mail: swonkeun@hallym.or.kr

() The Korean Society for Laboratory Medicine This is an Open Access article distributed under the terms of the Creative Commons Attribution Non-Commercial License (http://creativecommons.org/licenses/by-nc/4.0) which permits unrestricted non-commercial use, distribution, and reproduction in any medium, provided the original work is properly cited.

\section{INTRODUCTION}

The emergence of carbapenemase-producing Enterobacteriaceae (CPE) is a major clinical concern because these bacteria are resistant to multiple classes of antibiotics, which can lead to therapeutic failure [1]. CPE produce enzymes that fall into three classes according to the Ambler classification: class A $\beta$-lactamases (Klebsiella pneumoniae carbapenemase [KPC]), class B metallo- $\beta$ - 
lactamases (New Delhi metallo- $\beta$-lactamase [NDM], imipenemase [IMP], and Verona integron-encoded metallo- $\beta$-lactamase [VIM]), and class D $\beta$-lactamases (oxacillinase [OXA]-48). The carbapenemase genes in Enterobacteriaceae have been shown to be associated with mobile genetic elements such as plasmids or transposons, thereby facilitating infection outbreaks [2].

The first strain of KPC-producing K. pneumoniae was identified in North Carolina, USA, in 1996 [3]. Several outbreaks associated with these strains have been reported in the USA, South America, Europe, and China [4]. After the first identification of an OXA-48-producing $K$. pneumoniae strain in Istanbul, Turkey, in 2001 [5], numerous outbreaks caused by strains like OXA-48 have been reported in Europe, the Indian subcontinent, the Middle East, and Northern Africa [6]. In the present study, we report the prevalence and molecular epidemiology of CPE isolates collected from five university hospitals in Korea in 2015.

\section{METHODS}

\section{Study design}

From January to December 2015, five university hospitals in a central province (two hospitals in Seoul, two hospitals in Gyeonggi, and one hospital in Gangwon) of Korea collected prospectively ertapenem-nonsusceptible Enterobacteriaceae isolates including species such as Escherichia coli, Klebsiella pneumoniae, Enterobacter cloacae, Enterobacter aerogenes, Serratia marcescens, and Citrobacter freundii by means of a Vitek 2 (bioMérieux Vitek, Hazelwood, MO, USA) or MicroScan system (Siemens, Sacramento, CA, USA). The isolates were included in the study, if they were not susceptible to ertapenem (minimal inhibitory concentration [MIC] $>0.5 \mu \mathrm{g} / \mathrm{mL}$ ) [7]. This study protocols was approved by the Institutional Review Board of each institution, which decided to waive the informed consent.

\section{Genotypic detection of $\beta$-lactamase genes}

All ertapenem-nonsusceptible isolates were tested for carbapenemase by multiplex PCR. The carbapenemase gene was detected by PCR primers encompassing the entire coding region of genes blakpc, blandm, bla Imp, blavim, blages, and blaoxA-48 [8]. All carbapenemase-positive isolates were tested for extended-spectrum $\beta$-lactamase (ESBL) and plasmid-mediated AmpC genes by PCR according to previously described methods [9, 10]. All PCR products were directly sequenced with an automatic sequencer (model 373xl; Applied Biosystems, Weiterstadt, Germany).

\section{Antimicrobial susceptibility testing}

Antimicrobial susceptibility was tested in two hospitals that experienced an outbreak for 24 KPC-2 K. pneumoniae in hospital $B$ and 45 OXA-232 K. pneumoniae in hospital C. The MICs of the CPE isolates were determined by using an Etest (bioMérieux, Marcy-l'Etoile, France). The antimicrobial agents tested were: cefotetan, cefotaxime, ceftazidime, cefepime, aztreonam, ertapenem, imipenem, meropenem, amikacin, ciprofloxacin, tigecycline, and colistin. When available, the MIC results were interpreted according to the CLSI criteria, 2014 [7]. For tigecycline and colistin, the European Committee for Antimicrobial Susceptibility Testing (EUCAST) criteria (http://www.eucast.org/clinical_ breakpoints, January 2014) were used.

4. Molecular typing by pulsed-field gel electrophoresis (PFGE) Genetic relatedness of KPC-2 K. pneumoniae and OXA-232 K. pneumoniae isolates was collectively evaluated by PFGE at one time. Plugs containing Xbal-digested genomic DNA were prepared, and DNA fragments were separated for $20 \mathrm{hr}$ at $6 \mathrm{~V} / \mathrm{cm}$ at $11^{\circ} \mathrm{C}$ on a CHEF-DRII System (Bio-Rad, Hercules, CA, USA) with initial and final pulse times of $0.5 \mathrm{sec}$ and $30 \mathrm{sec}$, respectively. A lambda ladder (Bio-Rad) was used as a DNA size marker. Gels with PFGE-separated fragments of chromosomal DNA were blotted onto nylon membranes (Bio-Rad) and hybridized with probes by using the DIG DNA Labeling and Detection Kit (Roche Diagnostics GmbH, Mannheim, Germany). Similarity coefficients were calculated from Dice coefficients. Cluster analysis was conducted by the unweighted pair group method with arithmetic averages (UPGMA). Isolates that had a PFGE profile with more than $90 \%$ similarity (pulsotype [PT]) were considered closely related strains.

\section{Patient characteristics}

The clinical characteristics collected from patients who contracted KPC-2 K. pneumoniae and OXA-232 K. pneumoniae isolates were age, sex, sampling date, isolation site, hospitalization ward, hospitalization days, and in-hospital death by reviewing the medical records.

\section{Statistical analysis}

All calculations were performed using R software, version 3.2.4 (R Development Core Team 2016; http://www.R-project.org/). Categorical variables were compared by Chi-square test or Fisher's exact test, and continuous variables were compared by Student's t-test. All tests were two-sided, and differences with $P$ value $\leq 0.05$ were considered significant. 


\section{RESULTS}

\section{Distribution of CPE isolates}

Between January and December 2015, 393 (3.0\% of all 13,005 Enterobacteriaceae isolates) ertapenem-nonsusceptible, nonduplicated isolates were collected. These 393 isolates were 177 K. pneumoniae isolates (45.0\%), 108 Enterobacter cloacae isolates (27.5\%), 46 S. marcescens isolates (11.7\%), 36 Escherichia coli isolates (9.2\%), 21 Enterobacter aerogenes isolates (5.3\%), and five $C$. freundii isolates (1.3\%) (Table 1 ).

The distribution of different carbapenemases among the Enterobacteriaceae isolates is summarized in Table 2. Carbapenemases were responsible for resistance in 79 isolates $(0.6 \%$ of all 13,005 Enterobacteriaceae, $20.1 \%$ of all 393 ertapenem-resistant Enterobacteriaceae). Among the $79 \mathrm{CPE}$ isolates, 47 (59.5\%), 27 (34.2\%), 4 (5.1\%), and 1 (1.2\%) were OXA-232, KPC-2, IMP-1, and NDM-1 producers, respectively. All CPE isolates co-produced ESBLs; for example, KPC-2 K. pneumoniae isolates also had CTX-M-65, and OXA-232 K. pneumoniae isolates also had CTX-M-15. The most prevalent species among the OXA-232 and the KPC-2 strains were K. pneumoniae (89.8\%: OXA-232 at 58.2\% and KPC-2 at 31.6\%). Two hospitals experienced an outbreak (hospital B: KPC-2 K. pneumoniae, hospital C: OXA-232 K. pneumoniae), and no CPE were isolated in hospital D.

\section{Patient characteristics}

Fifty-three patients $(76.8 \%)$ were male. The mean age of the patients was $64.6 \mathrm{yr}$. The following specimens were provided: respiratory secretions $(n=31,44.9 \%)$, urine $(n=17,24.6 \%)$, wound or pus ( $n=9,13.1 \%)$, and blood $(n=5,7.3 \%)$. No CPE was isolated from outpatients. Forty-nine percent $(n=34)$ of isolates were from patients in intensive care units (ICU). All isolates were collected from patients after more than two days of hospitalization. The median number of hospitalization days before CPE isolation from the patients with KPC-2 and OXA-232 isolates was 40 and 16 days $(P=0.006)$, respectively. Twenty-eight percent $(n=19)$ of patients died during hospitalization (Table 3 ).

\section{Antimicrobial susceptibilities}

One-hundred percent and 92\% of KPC-2 K. pneumoniae isolates were susceptible to tigecycline and colistin, respectively, while 100\% were resistant to all cephalosporins, aztreonam, carbapenems, amikacin, and ciprofloxacin. Seventy-eight percent and $89 \%$ of OXA-232 K. pneumoniae isolates were susceptible to tigecycline and colistin, respectively. More than $90 \%$

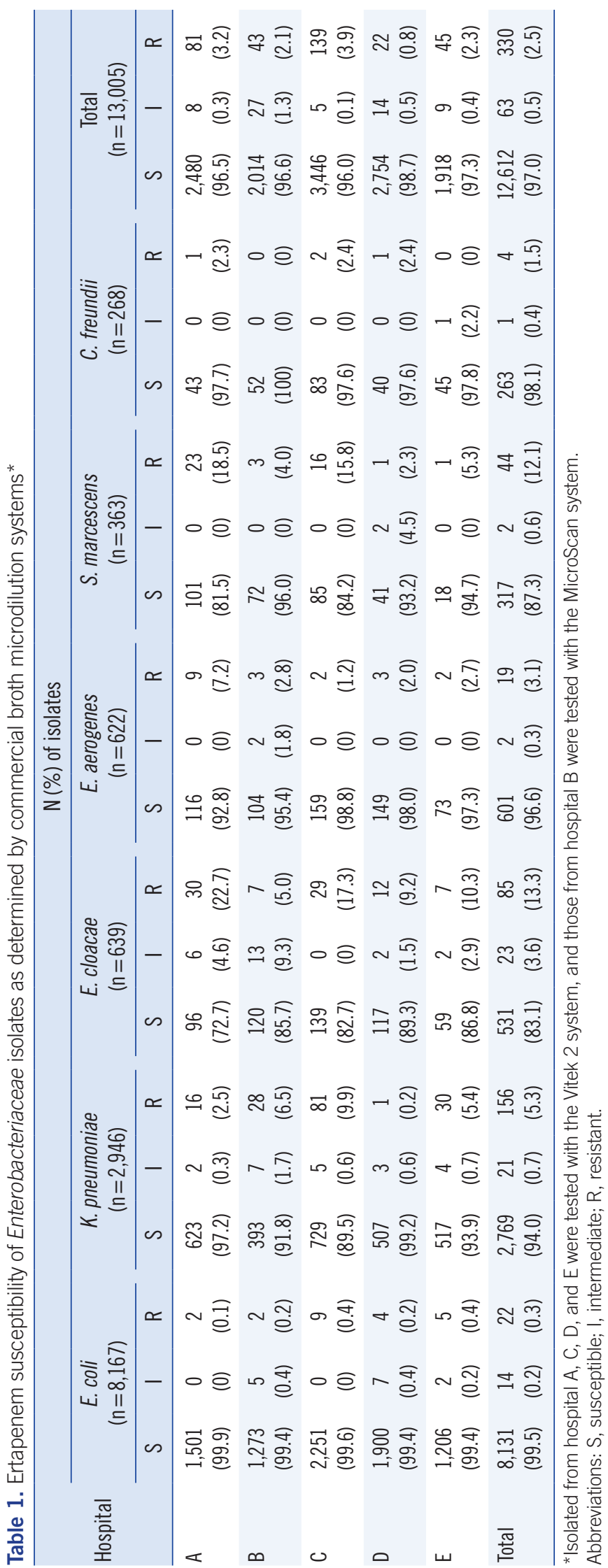


Table 2. Distribution of carbapenemase-producing Enterobacteriaceae isolates per hospital

\begin{tabular}{llcccccc}
\hline \multirow{2}{*}{ Carbapenemase } & \multirow{2}{*}{ Organism } & \multicolumn{6}{c}{ N of carbapenemase-producing Enterobacteriaceae isolates* } \\
\cline { 3 - 8 } & & Hospital A & Hospital B & Hospital C & Hospital D & Hospital E & Total \\
\hline \multirow{2}{*}{ OXA-232 } & K. pneumoniae & 0 & 0 & 45 & 0 & 1 & 46 \\
& E. coli & 0 & 0 & 1 & 0 & 0 & 1 \\
& K. pneumoniae & 0 & 24 & 0 & 0 & 1 & 25 \\
IMP-1 & E. coli & 1 & 0 & 0 & 0 & 0 & 1 \\
& E. cloacae & 1 & 0 & 0 & 0 & 0 & 1 \\
NDM-1 & K. pneumoniae & 0 & 0 & 0 & 0 & 2 & 2 \\
Total & E. cloacae & 0 & 0 & 2 & 0 & 0 & 2 \\
\hline
\end{tabular}

*1 KPC-2 E. coli harbored SHV-12 + CTX-M-65, 1 KPC-2 E. cloacae harbored CTX-M-15 (hospital A); 24 KPC-2 K. pneumoniae harbored CTX-M-65 (hospital B); 45 OXA-232 K. pneumoniae harbored 42 CTX-M 15, 1 SHV-2, 1 SHV-12, and 1 SHV-38, respectively, 1 OXA-232 E. coli harbored CTX-M-15, 2 IMP1 E. cloacae harbored CTX-M-14, 1 NDM-1 E. coli harbored CTX-M-15 (hospital C); 1 OXA-232 K. pneumoniae harbored CTX-M-15, 1 KPC-2 K. pneumoniae harbored SHV-12 + CTX-M-15, 2 IMP-1 K. pneumoniae harbored 1 SHV-12 and 1 SHV-12+CTX-M-15, respectively (hospital E).

Abbreviations: OXA, oxacillinase; KPC, Klebsiella pneumoniae carbapenemase; IMP, imipenemase; NDM, New Delhi metallo- $\beta$-lactamase.

Table 3. Clinical characteristics of patients with KPC-2-producing K. pneumoniae and OXA-232-producing K. pneumoniae isolates

\begin{tabular}{|c|c|c|c|c|}
\hline \multirow{2}{*}{ Characteristic } & \multicolumn{3}{|c|}{$\mathrm{N}(\%)$ of patients } & \multirow{2}{*}{$P$ value } \\
\hline & Total $(n=69)$ & $\mathrm{KPC}-2(n=24)$ & $0 \times A-232(n=45)$ & \\
\hline Age $($ mean $\pm S D)$ & $64.6 \pm 11.8$ & $66.9 \pm 8.6$ & $63.4 \pm 13.1$ & 0.189 \\
\hline Male gender & $53(76.8)$ & $19(79.2)$ & $34(75.6)$ & 0.969 \\
\hline Specimen & & & & 0.059 \\
\hline Respiratory & $31(44.9)$ & $6(25.0)$ & $25(55.6)$ & \\
\hline Urine & $17(24.6)$ & $8(33.4)$ & $9(20.0)$ & \\
\hline Wound or pus & $9(13.1)$ & $6(25.0)$ & $3(6.7)$ & \\
\hline Blood & $5(7.3)$ & $2(8.3)$ & $3(6.7)$ & \\
\hline Others & $7(10.1)$ & $2^{*}(8.3)$ & $5^{\dagger}(11.0)$ & \\
\hline ICU hospitalization & $34(49.3)$ & $11(45.8)$ & $23(51.1)$ & 0.869 \\
\hline \multicolumn{5}{|c|}{ Hospitalization days [median (range)] } \\
\hline Before CPE isolation & $17(1-187)$ & $40(2-142)$ & $16(1-187)$ & 0.006 \\
\hline After CPE isolation & $21(1-132)$ & $30(3-124)$ & $12(1-132)$ & 0.142 \\
\hline Died during hospitalization & $19(27.5)$ & $5(20.8)$ & $14(31.1)$ & 0.486 \\
\hline
\end{tabular}

*These two isolates were recovered from bile juice; ${ }^{\dagger}$ These five isolates were recovered from catheter tips $(n=3)$, ascites $(n=1)$, and bile juice $(n=1)$.

Abbreviations: KPC, Klebsiella pneumoniae carbapenemase; OXA, oxacillinase; ICU, intensive care unit; CPE, carbapenemase-producing Enterobacteriaceae.

of them were resistant to all cephalosporins, aztreonam, ertapenem, meropenem, and ciprofloxacin, but the imipenem resistance rate was $69 \%$ (Table 4).

\section{Pulsed-field gel electrophoresis typing}

Of the 24 KPC-2 K. pneumoniae and 45 OXA-232 K. pneumoniae isolates, 61 were available for PFGE. This analysis revealed a unique pattern for $20 \mathrm{KPC}-2 \mathrm{~K}$. pneumoniae isolates in hospital B (Fig. 1). PFGE of 41 OXA-232 K. pneumoniae isolates from hospital $\mathrm{C}$ identified seven PTs, of which 21 isolates belonged to the dominant PT1, and nine were PT2 (Fig. 2).

\section{DISCUSSION}

Until now, the carbapenem resistance rate among Enterobacteriaceae isolates from Korea has been relatively low and stable [11]. The present study showed that the incidence of ertapenem-nonsusceptible Enterobacteriaceae is 3\% and the incidence of CPE is $0.6 \%$ (out of 13,005 isolates) of Enterobacteriaceae and $20.1 \%$ (out of 383 isolates) of ertapenem-nonsusceptible Enterobacteriaceae. The most common CPE organism was $K$. pneumoniae (92.4\%). The overall incidence of carbapenem-resistant Enterobacteriaceae (CRE) in the USA is estimated to be 
Jeong SH, et al.

Carbapenemase-producing Enterobacteriaceae in Korea

Table 4. Antimicrobial susceptibility of carbapenemase-producing K. pneumoniae isolates

\begin{tabular}{|c|c|c|c|c|c|c|c|c|}
\hline \multirow{3}{*}{ Antimicrobial agent } & \multicolumn{4}{|c|}{ KPC-2-producing K. pneumoniae isolates $(n=24)$} & \multicolumn{4}{|c|}{ OXA-232-producing K. pneumoniae isolates $(n=45)$} \\
\hline & \multicolumn{3}{|c|}{$\operatorname{MIC}(\mu \mathrm{g} / \mathrm{mL})$} & \multirow{2}{*}{$\% R$} & \multicolumn{3}{|c|}{ MIC $(\mu \mathrm{g} / \mathrm{mL})$} & \multirow{2}{*}{$\% R$} \\
\hline & Range & $\mathrm{MIC}_{50}$ & $\mathrm{MIC}_{90}$ & & Range & $\mathrm{MIC}_{50}$ & $\mathrm{MIC}_{90}$ & \\
\hline Cefotetan & $128->256$ & $>256$ & $>256$ & 100 & $1->256$ & 128 & 256 & 89 \\
\hline Cefotaxime & $>32$ & $>32$ & $>32$ & 100 & $1->32$ & $>32$ & $>32$ & 98 \\
\hline Ceftazidime & $128->256$ & 256 & $>256$ & 100 & $0.25->256$ & $>256$ & $>256$ & 96 \\
\hline Cefepime & $128->256$ & $>256$ & $>256$ & 100 & $0.25->256$ & $>256$ & $>256$ & 93 \\
\hline Aztreonam & $>256$ & $>256$ & $>256$ & 100 & $<0.125->256$ & $>256$ & $>256$ & 93 \\
\hline Ertapenem & $>32$ & $>32$ & $>32$ & 100 & $1->32$ & $>32$ & $>32$ & 96 \\
\hline Imipenem & $32->32$ & $>32$ & $>32$ & 100 & $0.5->32$ & 4 & 16 & 69 \\
\hline Meropenem & $>32$ & $>32$ & $>32$ & 100 & $0.5->32$ & 16 & $>32$ & 96 \\
\hline Amikacin & $>256$ & $>256$ & $>256$ & 100 & $2->256$ & $>256$ & $>256$ & 84 \\
\hline Ciprofloxacin & $>32$ & $>32$ & $>32$ & 100 & $<0.125->32$ & $>32$ & $>32$ & 93 \\
\hline Tigecycline & $0.25-2$ & 0.5 & 1 & 0 & $0.5-4$ & 2 & 4 & 22 \\
\hline Colistin & $0.126-16$ & 0.25 & 0.5 & 8 & $0.125-32$ & 0.5 & 4 & 11 \\
\hline
\end{tabular}

Abbreviations: KPC, Klebsiella pneumoniae carbapenemase; OXA, oxacillinase; MIC, minimum inhibitory concentration; $\mathrm{MIC}_{50}$, minimum inhibitory concentration for $50 \%$ of isolates; $\mathrm{MIC}_{90}$, minimum inhibitory concentration for $90 \%$ of isolates; $\% \mathrm{R}$, \% of resistance.

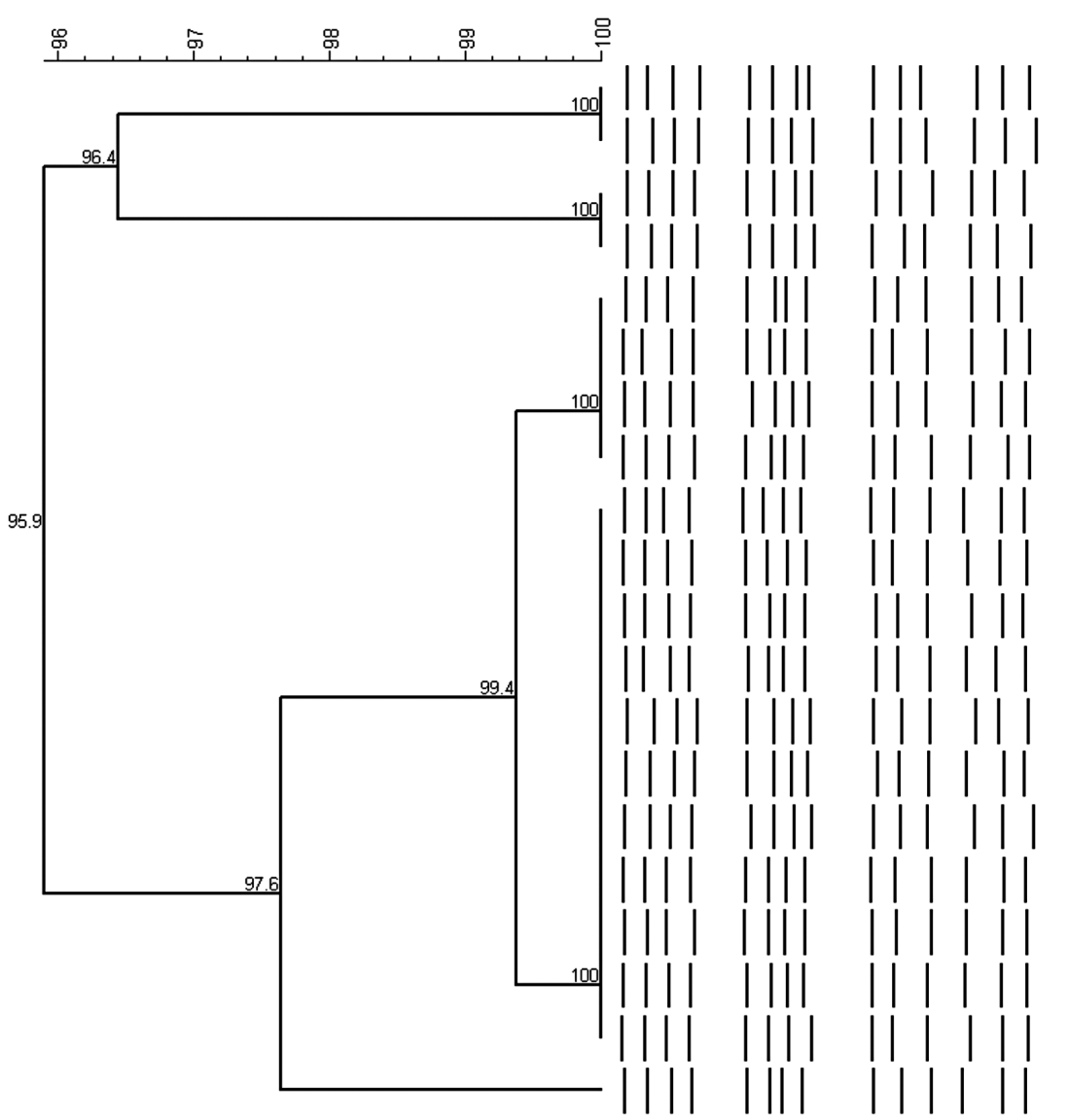

\begin{tabular}{|c|c|c|c|}
\hline Isolate No. & Pulsotype & $\begin{array}{l}\text { Month-day of } \\
\text { collection }\end{array}$ & Ward \\
\hline B-222 & PT1 & $12-28$ & Internal medicine \\
\hline B-223 & PT1 & $12-28$ & Internal medicine \\
\hline B-210 & PT1 & $10-13$ & Surgical ICU \\
\hline B-220 & PT1 & $12-25$ & Otolaryngology \\
\hline B-199 & PT1 & $09-08$ & Surgical ICU \\
\hline B-184 & PT1 & $08-18$ & Internal medicine \\
\hline B-200 & PT1 & $09-11$ & Internal medicine \\
\hline B-180 & PT1 & $08-11$ & Neurosurgery \\
\hline B-154 & PT1 & $06-04$ & Surgical ICU \\
\hline B-189 & PT1 & $08-20$ & Surgical ICU \\
\hline B-190 & PT1 & $08-26$ & Internal medicine \\
\hline B-194 & PT1 & $08-28$ & Surgical ICU \\
\hline B-212 & PT1 & $10-22$ & Neurosurgery \\
\hline B-216 & PT1 & $11-18$ & Internal medicine \\
\hline B-218 & PT1 & $11-23$ & Surgical ICU \\
\hline B-168 & PT1 & $07-11$ & Surgical ICU \\
\hline B-173 & PT1 & $07-25$ & Neurosurgery \\
\hline B-174 & PT1 & $07-27$ & Neurosurgery \\
\hline B-188 & PT1 & $08-20$ & Surgical ICU \\
\hline B-161 & PT1 & $06-25$ & Medical ICU \\
\hline
\end{tabular}

Fig. 1. Pulsed-field gel electrophoresis (PFGE) patterns of KPC-2-producing K. pneumoniae isolated in hospital B ( $n=20)$. Isolates that exhibited PFGE dendrograms with more than 90\% similarity were considered as one pulsotype (PT).

Abbreviations: KPC, Klebsiella pneumoniae carbapenemase; ICU, intensive care unit. 


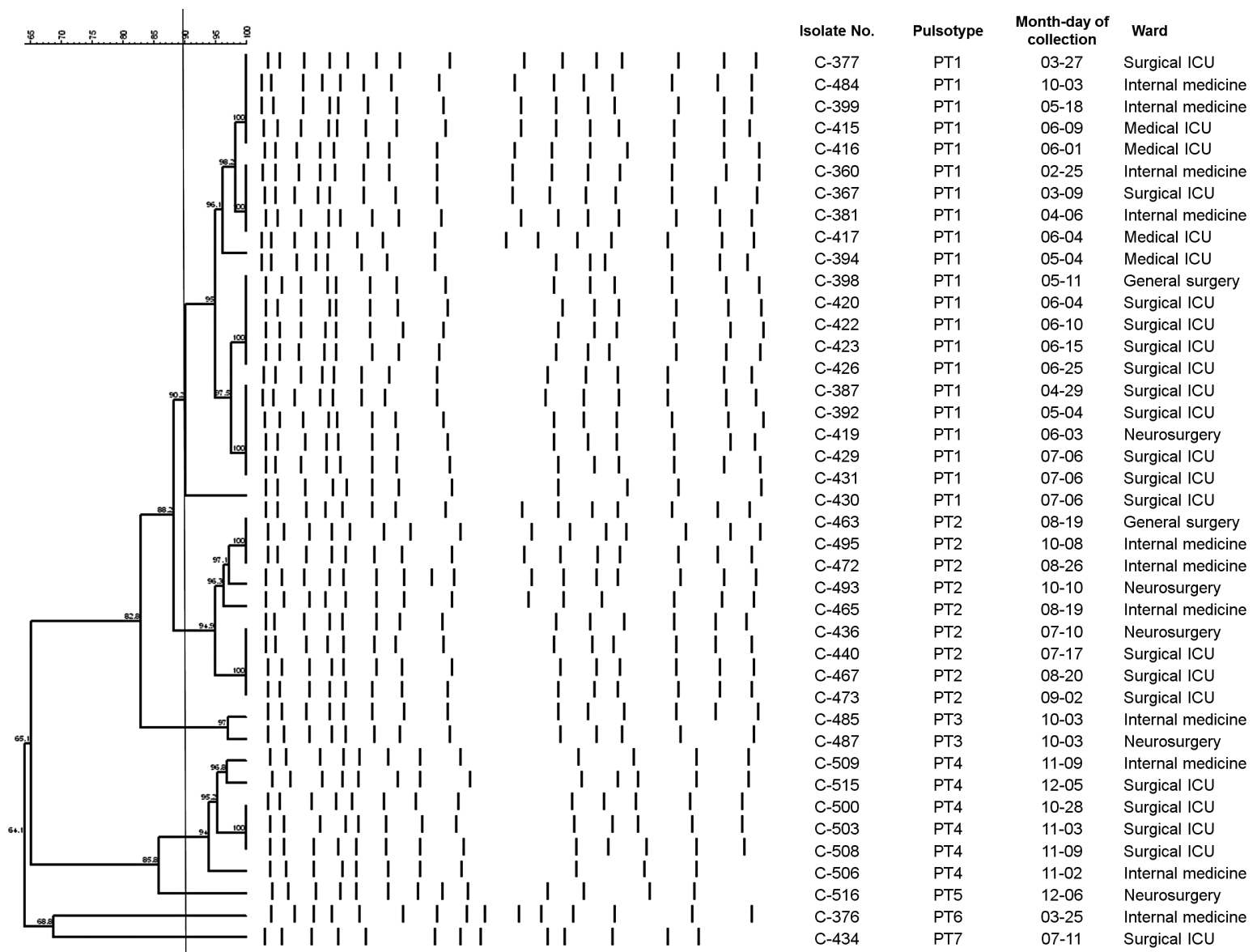

Fig. 2. Pulsed-field gel electrophoresis (PFGE) patterns of OXA-232-producing $K$. pneumoniae isolated in hospital $\mathrm{C}$ ( $\mathrm{n}=41$ ). Isolates that exhibited PFGE dendrograms with more than $90 \%$ similarity were considered as one pulsotype (PT). Black line in dendrogram represents percentage similarity cut-off.

Abbreviations: OXA, oxacillinase; ICU, intensive care unit.

$1.4 \%$ to $4.2 \%$ [12]. CPE have been detected in $81.7 \%$ and $20 \%$ of CRE isolates in the USA [13] and Taiwan [14], respectively.

Three main carbapenemases are reported worldwide: KPC, NDM, and OXA-48-like. KPC strains are mostly found in the USA, Israel, Greece, and Italy. The Indian subcontinent is recognized as an NDM and OXA-48-like endemic zone. OXA-48like is often seen in the Mediterranean area and Northern Africa [4]. OXA-162, -163, -181, -204, and -232 were identified as OXA-48 variants [6]. Korea is known for outbreaks of $K$. pneumoniae that produce KPC-2, NDM-1, and OXA-232 [15-17]. In the present study, two of five hospitals had an outbreak (hospital B: KPC-2 K. pneumoniae, hospital C: OXA-232 K. pneumoniae), and no CPE were isolated in one hospital. All CPE isolates Coproduced ESBLs: mainly CTX-M-65 and CTX-M-15.

Most patients in this study were older male inpatients, and the most common specimens analyzed were respiratory secretions and urine. These findings are similar to the results of other studies [14, 18, 19]. The median number of hospitalization days before $\mathrm{CPE}$ isolation among the patients with these isolates was 17 days, and the median number of hospitalization days of patients with KPC-2 isolates was 40 days. Long-term hospitalization may play an important role in the spread of CPE. This study excluded analysis of infection and colonization with CPE because it was hard to distinguish some cases retrospectively.

In this study, all KPC-2 K. pneumoniae isolates were highly resistant to all cephalosporins, aztreonam, and carbapenems. More than 90\% of OXA-232 K. pneumoniae isolates were resistant to all cephalosporins, aztreonam, ertapenem, and meropenem. The likely reason is that all OXA-232 K. pneumoniae isolates also produce ESBLs. OXA-48 hydrolyzes penicillins effectively, but it only weakly hydrolyzes carbapenems. In addition, this enzyme shows very weak activity toward extended-spectrum cephalosporins [20]. OXA-48-like producers that do not produce any ESBLs are still susceptible to broad-spectrum cepha- 
losporins and can be susceptible or resistant to carbapenems [6]. Only tigecycline and colistin remained effective against most, but not all, KPC-2 and OXA-232 K. pneumoniae isolates.

The PFGE analysis revealed a unique pattern for $20 \mathrm{KPC}-2 \mathrm{~K}$. pneumoniae and 41 OXA-232 K. pneumoniae isolates, of which 21 isolates belonged to the dominant PT1, and nine were PT2. These findings highlighted the risk of clonal dissemination of KPC-2 K. pneumoniae and OXA-232 K. pneumoniae in certain wards, especially in ICUs.

In summary, CPE strains are present in Korea, with the main K. pneumoniae isolates producing OXA-232 and KPC-2. Interestingly, the prevalence and predominant genotypes of CPE in Korea showed hospital-specific differences such as epidemic presence in two hospitals, sporadic presence in two hospitals, and absence in one hospital. These findings indicate that CPE dissemination is at an early stage in Korea. Therefore, greater efforts to control the nosocomial spread of CPE are warranted. Our results were based on isolates from five university hospitals. Among the strains, most were derived from two hospitals. This situation does not reflect the general epidemiology of CPE in Korea; hence, further large-scale research including isolates from acute care and long-term care hospitals is needed.

\section{Authors' Disclosures of Potential Conflicts of Interest}

No potential conflicts of interest relevant to this article were reported.

\section{Acknowledgments}

This study was supported by a research grant from the Korean Health Technology R\&D Project, Ministry of Health \& Welfare, Republic of Korea (HI12C0756).

\section{REFERENCES}

1. Tzouvelekis LS, Markogiannakis A, Psichogiou M, Tassios PT, Daikos GL. Carbapenemases in Klebsiella pneumoniae and other Enterobacteriaceae: an evolving crisis of global dimensions. Clin Microbiol Rev 2012; 25:682-707.

2. Queenan AM and Bush K. Carbapenemases: the versatile $\beta$-lactamases. Clin Microbiol Rev 2007;20:440-58.

3. Yigit H, Queenan AM, Anderson GJ, Domenech-Sanchez A, Biddle JW, Steward CD, et al. Novel carbapenem-hydrolyzing $\beta$-lactamase, KPC-1, from a carbapenem-resistant strain of Klebsiella pneumoniae. Antimicrob Agents Chemother 2001;45:1151-61.
4. Nordmann P, Naas T, Poirel L. Global spread of Carbapenemase-producing Enterobacteriaceae. Emerg Infect Dis 2011;17:1791-8.

5. Poirel L, Héritier C, Tolün V, Nordmann P. Emergence of oxacillinasemediated resistance to imipenem in Klebsiella pneumoniae. Antimicrob Agents Chemother 2004;48:15-22.

6. Poirel L, Potron A, Nordmann P. OXA-48-like carbapenemases: the phantom menace. J Antimicrob Chemother 2012;67:1597-606.

7. Clinical and Laboratory Standards Institute. Performance standards for antimicrobial susceptibility testing. Twenty-fourth Informational supplement, M100-S24. Wayne, PA: Clinical and Laboratory Standards Institute, 2014

8. Poirel L, Walsh TR, Cuvillier V, Nordman P. Multiplex PCR for detection of acquired carbapenemase genes. Diagn Microbiol Infect Dis 2011;70: 119-23.

9. Ryoo NH, Kim EC, Hong SG, Park YJ, Lee K, Bae IK, et al. Dissemination of SHV-12 and CTX-M-type extended-spectrum $\beta$-lactamases among clinical isolates of Escherichia coli and Klebsiella pneumoniae and emergence of GES-3 in Korea. J Antimicrob Chemother 2005;56: 698-702.

10. Pérez-Pérez FJ and Hanson ND. Detection of plasmid-mediated AmpC $\beta$-lactamase genes in clinical isolates by using multiplex PCR. J Clin Microbiol 2002;40:2153-62.

11. Korea Centers for Disease Control and Prevention. Korean Antimicrobial Resistance Monitoring System (KARMS) annual report, 2014. Korea Centers for Disease Control and Prevention, 2015.

12. Centers for Disease Control and Prevention. Vital signs: carbapenem-resistant Enterobacteriaceae. MMWR Morb Mortal Wkly Rep 2013;62: 165-70.

13. Pollett S, Miller S, Hindler J, Uslan D, Carvalho M, Humphries RM. Phenotypic and molecular characteristics of carbapenem-resistant Enterobacteriaceae in a health care system in Los Angeles, California, from 2011-2013. J Clin Microbiol 2014;52:4003-9.

14. Tseng IL, Liu YM, Wang SJ, Yeh HY, Hsieh CL, Lu HL, et al. Emergence of carbapenemase producing Klebsiella pneumoniae and spread of KPC-2 and KPC-17 in Taiwan: a nationwide study from 2011 to 2013. PLoS One 2015;10:e0138471.

15. Kim MN, Yong D, An D, Chung HS, Woo JH, Lee K, et al. Nosocomial clustering of NDM-1-producing Klebsiella pneumoniae sequence type 340 strains in four patients at a South Korean tertiary care hospital. J Clin Microbiol 2012;50:1433-6.

16. Hong SK, Yong D, Kim K, Hong SS, Hong SG, Khosbayar T, et al. First outbreak of KPC-2-producing Klebsiella pneumoniae sequence type 258 in a hospital in South Korea. J Clin Microbiol 2013;51:3877-9.

17. Jeong SH, Lee KM, Lee J, Bae IK, Kim JS, Kim HS, et al. Clonal and horizontal spread of the blaoxA-232 gene among Enterobacteriaceae in a Korean hospital. Diagn Microbiol Infect Dis 2015;82:70-2.

18. Lefebvre B, Lévesque S, Bourgault AM, Mulvey MR, Mataseje L, Boyd D, et al. Carbapenem non-susceptible Enterobacteriaceae in Quebec, Canada: results of a laboratory surveillance program (2010-2012). PLoS One 2015;10:e0125076.

19. Brañas P, Villa J, Viedma E, Mingorance J, Orellana MA, Chaves F. Molecular epidemiology of carbapenemase-producing Klebsiella pneumoniae in a hospital in Madrid: Successful establishment of an OXA-48 ST11 clone. Int J Antimicrb Agents 2015;46:111-6.

20. Docquier JD, Calderone V, De Luca F, Benvenuti M, Giuliani F, Bellucci L, et al. Crystal structure of the OXA-48 $\beta$-lactamase reveals mechanistic diversity among class D carbapenemases. Chem Biol 2009;16:540-7. 